Received: 2 December 2016

Accepted: 19 December 2017

Published online: 17 January 2018

\title{
Endothelial nitric oxide synthase
} overexpressing human early outgrowth cells inhibit coronary artery smooth muscle cell migration through paracrine functions

\begin{abstract}
Sergio Guber, Talin Ebrahimian, Maryam Heidari, Nicoletta Eliopoulos \& Stephanie Lehoux
Cells mobilized from the bone marrow can contribute to endothelial regeneration and repair. Nevertheless, cardiovascular diseases are associated with diminished numbers and function of these cells, attenuating their healing potential. Gene transfer of endothelial nitric oxide synthase (eNOS) can restore the activity of circulating cells. Furthermore, estrogen accelerates the reendothelialization capacity of early outgrowth cells (EOCs). We hypothesized that overexpressing eNOS alone or in combination with estrogen stimulation in EOCs would potentiate the beneficial effects of these cells in regulating smooth muscle cell (SMC) function. Native human EOCs did not have any effect on human coronary artery SMC ( $\mathrm{hCASMC}$ ) proliferation or migration. Transfecting EOCs with a human eNOS plasmid and/or stimulating with 173 -estradiol $\left(E_{2}\right)$ increased NO production 3-fold and enhanced EOC survival. Moreover, in co-culture studies, eNOS overexpressing or E2-stimulated EOCs reduced hCASMC migration (by $23 \%$ and $56 \%$ respectively), vs. control EOCs. These effects do not implicate ERK1/2 or focal adhesion kinases. Nevertheless, NOS-EOCs had no effect on hCASMC proliferation. These results suggest that overexpressing or activating eNOS in EOCs increases their survival and enhances their capacity to regulate SMC migration through paracrine effects. These data elucidate how eNOS overexpression or activation in EOCs can prevent vascular remodeling.
\end{abstract}

Endothelial damage and dysfunction contribute to the development of atherosclerosis, coronary heart disease and restenosis after angioplasty. Endothelial cells (ECs) usually remain quiescent as a result of contact inhibition. However, with age or after mechanical injury, cells may detach and die or undergo apoptosis, facilitating atherosclerotic lesion formation ${ }^{1}$. Loss of EC function also promotes changes in vascular smooth muscle cell (SMC) proliferation, migration and extracellular matrix production ${ }^{2}$. This issue is a prominent feature of restenosis after angioplasty. Although stents are commonly coated with anti-proliferative agents to hamper the excessive proliferation and migration of smooth muscle cells, these drugs also slow vascular reendothelialization, in part by promoting early outgrowth cell (EOC) apoptosis ${ }^{3}$.

EOCs are premature circulating cells that are derived from the bone marrow and are involved in postnatal vasculogenesis and reendothelialization after endothelial damage $e^{4-6}$. EOCs are capable of exerting paracrine functions and differentiating into functional endothelial cells. We and others have shown that EOCs can be recruited to sites of endothelial injury and participate in reendothelialization ${ }^{7-9}$. The therapeutic significance of EOCs is limited because of their low numbers in circulation, particularly in patients with elevated cardiovascular disease risk $^{10}$. Nevertheless, the number and function of circulating EOCs may be influenced favorably by factors such as estrogen levels and statin therapy. Estrogen treatment causes EOCs to secrete more growth factors and facilitates their integration at sites of angiogenesis ${ }^{11}$. Both estrogen and statins have also been shown to act through 

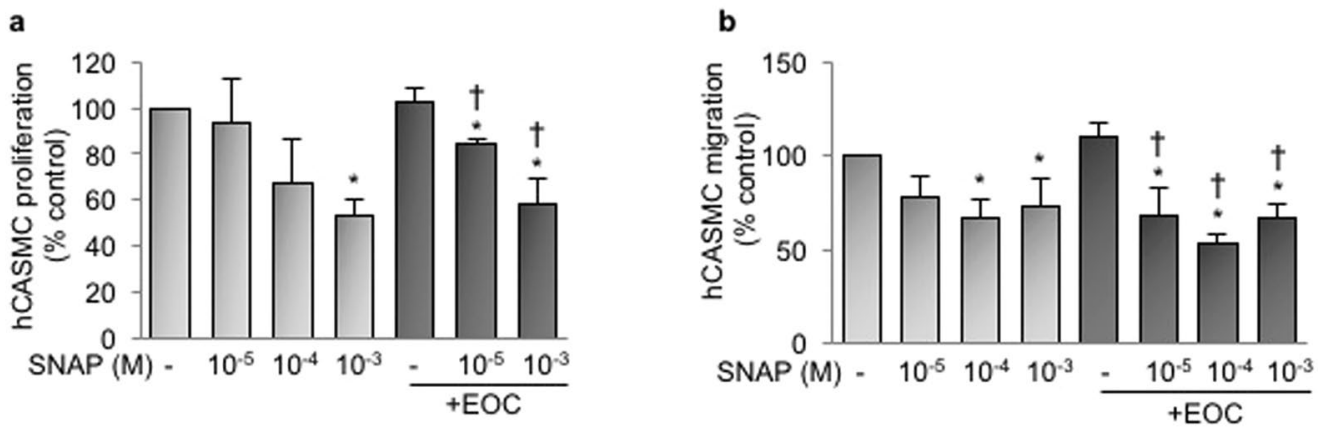

c
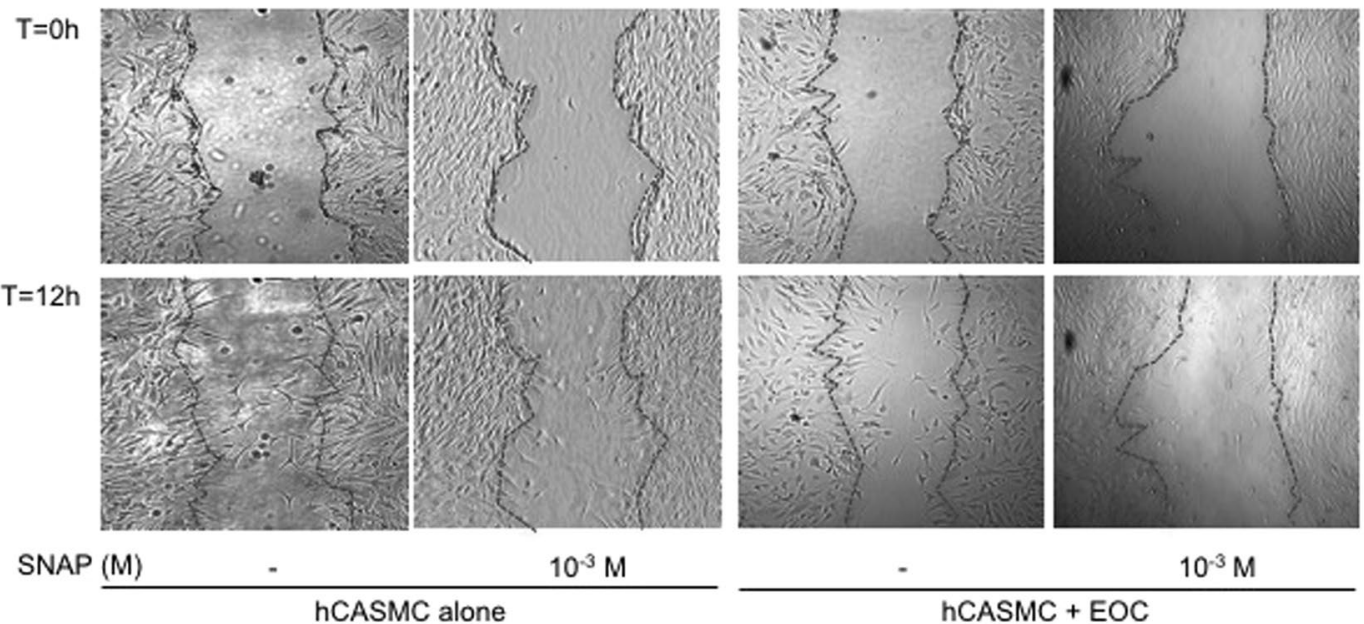

Figure 1. Intact EOCs do not affect SMC proliferation or migration. Human coronary artery smooth muscle cells (hCASMCs), co-cultured or not with early outgrowth cells (EOCs), were stimulated with S-nitroso-Nacetylpenicillamine (SNAP) at different concentrations as indicated. (a) hCASMC proliferation was assessed by $24 \mathrm{~h}$ BrdU incorporation. (b) hCASMC migration was assessed by scratch assay after $12 \mathrm{~h}$. Representative images are shown in (c). Data are mean \pm SEM of $n=6$, expressed as \% control (hCASMCs cultured alone, in absence of SNAP treatment $(-))$. $* \mathrm{p}<0.05$ vs untreated $(-), \dagger<0.05$ vs untreated + EOCs.

the eNOS/Akt pathway ${ }^{12,13}$. In the case of estrogen, these effects may involve both genomic processes (increased eNOS transcription) and non-genomic processes (enhanced enzymatic activity of eNOS) ${ }^{12,14-16}$. Appropriately, the reendothelialization and neovascularization capacity of EOCs is increased when eNOS is upregulated ${ }^{17-19}$. Moreover, it was shown that in vivo transplantation of EOCs overexpressing eNOS in rats repairs injured vessels by inhibiting neointimal hyperplasia and restoring vascular function ${ }^{7}$. Importantly, two recent clinical trials reported the safety and potential efficacy of endothelial progenitor cells overexpressing eNOS and autologous CD34 positive cell treatments in patients with pulmonary arterial hypertension and left ventricular dysfunction post-STEMI, respectively ${ }^{20,21}$.

Surprisingly, although the beneficial effects of eNOS overexpressing EOCs has been well documented in vivo, there is little known on how these cells influence SMCs. We hypothesized that EOCs inhibit SMC proliferation and migration, and that eNOS overexpression would enhance EOC function. In our study, eNOS overexpression was performed either by transfecting EOCs with an eNOS plasmid or by stimulating EOCs with estrogen. We observed that eNOS-overexpressing or estrogen-stimulated EOCs have an increased survival and inhibit migration of SMCs through paracrine effects.

\section{Results}

EOCs do not affect human coronary artery SMC (hCASMC) proliferation or migration. Nitric oxide has been shown to decrease proliferation in cultured rat $\mathrm{SMCs}^{22}$. To determine if EOCs have a similar effect, human coronary artery SMCs (hCASMCs) were treated with the NO donor S-nitroso-N-acetylpenicillamine (SNAP, $10^{-5}-10^{-3} \mathrm{M}$ ) and/or co-cultured with $10^{6}$ EOCs. EOCs were seeded in cell culture well inserts, such that any effects on SMCs would be through paracrine stimulation rather than direct contact. Proliferation was assessed by $24 \mathrm{~h} \mathrm{BrdU}$ incorporation in hCASMCs. A scratch assay was performed to evaluate hCASMC migration after $8 \mathrm{~h}$. EOCs alone did not have any effect on SMC proliferation or migration (Fig. 1a,b). On the contrary, SNAP decreased the proliferation and migration of SMCs in a dose dependent manner. Nevertheless, hCASMC sensitivity to SNAP was accrued when SNAP was administered in the presence of EOCs. 
a
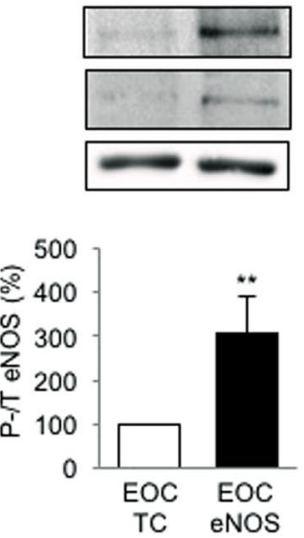

c

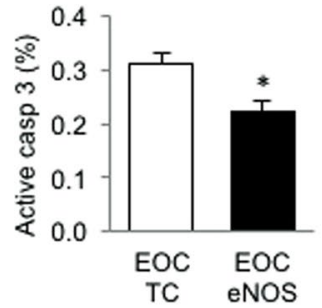

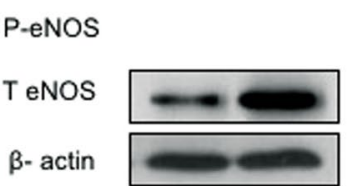

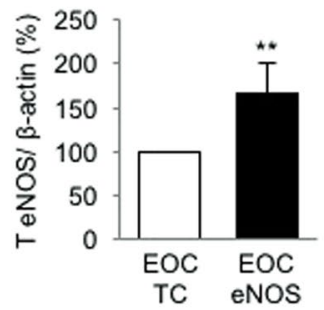

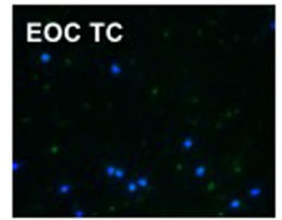
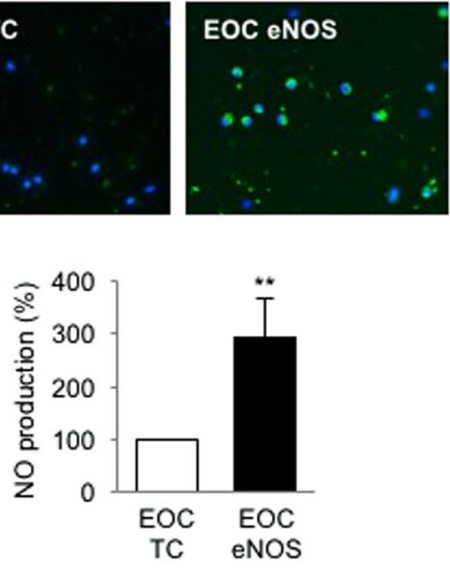

Figure 2. Transfection of EOCs with eNOS plasmid increases NO production and cell survival. Human EOCs were transfected with a transfection control plasmid (EOC TC) or eNOS plasmid (EOC eNOS) for 48 hours. (a) Total (T) and phosphorylated (pS1177) (P) eNOS expression was evaluated by western blot. (b) Nitric oxide (NO) production was assessed by DAF-FM diacetate, an NO fluorescent probe (green). (c) Apoptosis was measured by active caspase 3 flow cytometry. (d) The Bcl-2/Bax protein ratio was assessed by western blot and expressed as $\%$ of EOC TC. Data are mean \pm SEM of $n=6, * p<0.05$ and $* * p<0.01$ vs EOC TC.

Transfection of EOCs with eNOS plasmid increases NO production and cell survival. In order to improve the functionality of EOCs, we chose to up-regulate NO synthesis in the cells. Human EOCs were transfected with either eNOS or control plasmid for 48 hours. Both total expression levels of eNOS and eNOS phosphorylation at serine 1177 were increased 3-fold the eNOS-transfected EOCs compared with control transfected cells (Fig. 2a). Accordingly, we also observed a significant increase of NO production in cells transfected with eNOS plasmid (Fig. 2b). Moreover, because NO is a protective factor, we assessed EOC apoptosis and survival. We found a significant decrease in active caspase 3 expression, which is a pro-apoptotic factor (Fig. 2c), and an increase in the Bcl-2/ Bax expression ratio, a molecular index of cell survival, in eNOS-transfected EOCs compared with control transfected cells (Fig. 2d). Analysis of annexin V/propidium iodide staining by flow cytometry further revealed a $17 \pm 7 \%$ reduction in cell apoptosis among EOC eNOS cells compared with EOC TC. These results suggest that eNOS plasmid transfection of EOCs not only upregulates NO production but also increases their survival.

eNOS-transfected EOCs decrease migration but not proliferation of hCASMCs. To test whether eNOS overexpressing EOCs regulate hCASMC migration and proliferation, hCASMCs were co-cultured with EOCs transfected with eNOS or control plasmid. As seen in Fig. 3a (upper panel) and in confirmation of Fig. 1, there was no significant difference in hCASMC migration whether alone or in the presence of native EOCs. However, hCASMCs co-cultured with eNOS-overexpressing EOCs migrated less than hCASMCs co-cultured with transfection control EOCs (Fig. 3a, lower panel). These results suggest that NO released by eNOS-transfected EOCs reduces hCASMC migration through paracrine effects. In contrast, we did not observe any effect of eNOS-overexpressing EOCs on hCASMC proliferation (Fig. 3b).

Estrogen increases eNOS phosphorylationN, NO production and survival of EOCs. Since NO production has been shown to be regulated by estrogen stimulation, we used estrogen as an alternate approach to up-regulate NO production by EOCs. We started with a dose response study to determine the optimal estrogen $\left(\mathrm{E}_{2}\right)$ concentration to be used, and found that $10^{-9} \mathrm{M} \mathrm{E}_{2}$ induced the greatest NO production by EOCs (Fig. 4a). This concentration was used for all subsequent experiments. We observed a significant increase in eNOS phosphorylation (Fig. 4b) as well as Bcl-2/ Bax expression ratio (Fig. 4c) in EOCs stimulated with $\mathrm{E}_{2}$ compared with dimethysulfoxide vehicle (DMSO 1:4000). This suggests that $\mathrm{E}_{2}$ increases NO production by upregulating eNOS, leading to an increase of EOC survival. 


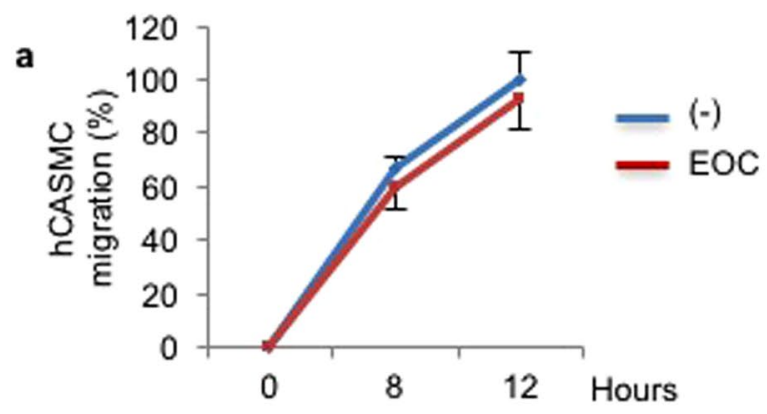

b
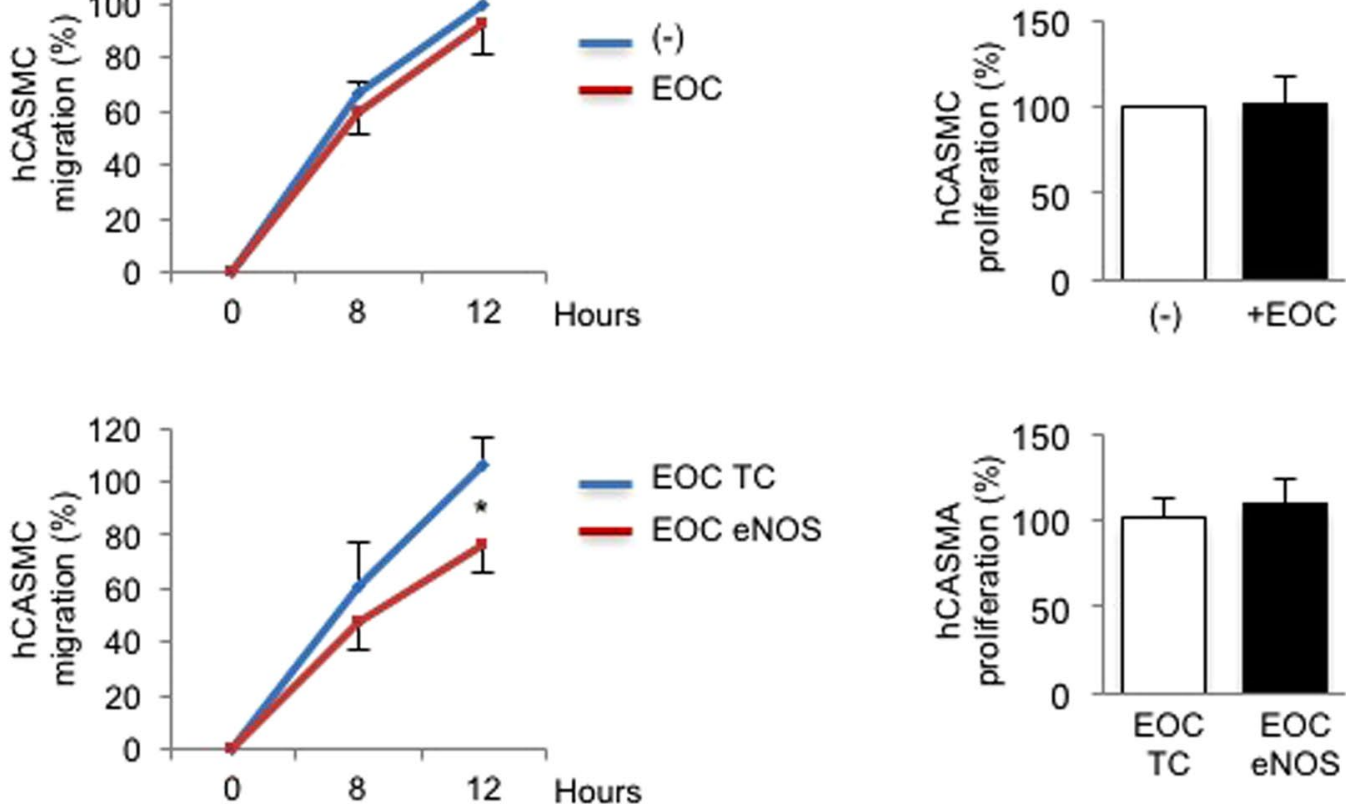

EOC TC

EOC ENOS

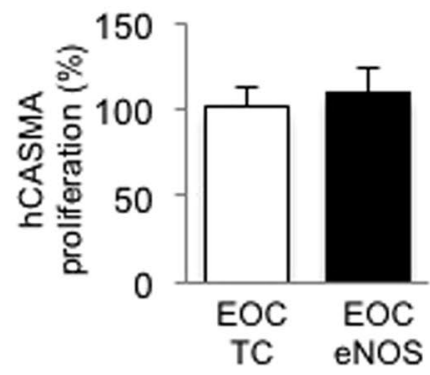

C
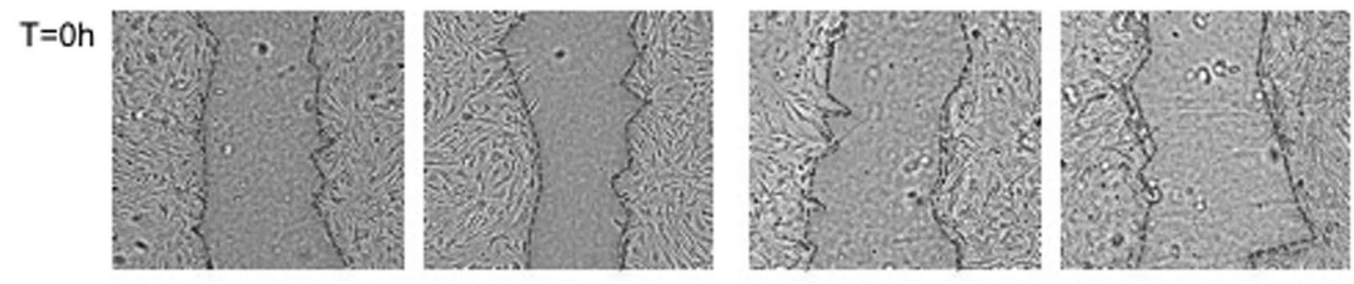

$\mathrm{T}=8 \mathrm{~h}$
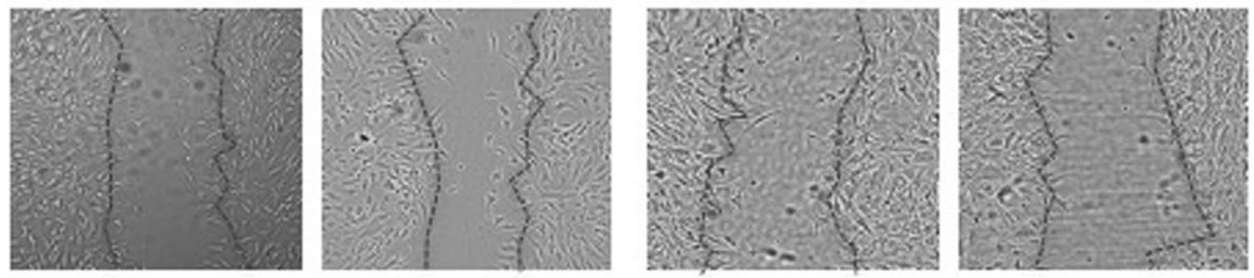

$\mathrm{T}=12 \mathrm{~h}$

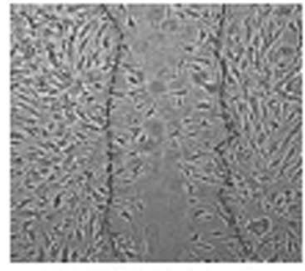

hCASMC alone

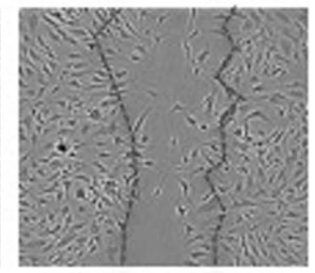

$h C A S M C+E O C$

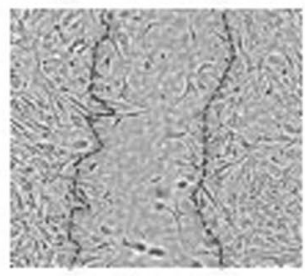

hCASMC + EOC TC

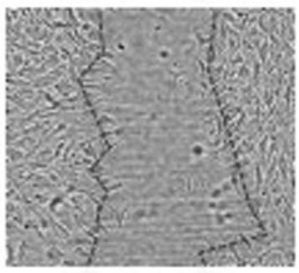

hCASMC + EOC ENOS

Figure 3. eNOS-transfected EOCs decrease migration but not proliferation of hCASMCs. Human coronary artery smooth muscle cells (hCASMC) were either cultured in the absence of EOCs ( - ) or co-cultured with human EOCs transfected with a transfection control plasmid (EOC TC) or eNOS plasmid (EOC eNOS) for up to 12 hours (a) or 48 hours (b). (a) hCASMC migration was assessed by scratch assay. Data are expressed as \% maximal migration observed in hCASMC cultured in the absence of EOCs. Representative images are shown in (c). (b) hCASMC proliferation was measured by Ki67 immunostaining as a ratio of total cell number. Data are expressed as \% maximal proliferation observed in hCASMC cultured in the absence of EOCs. Data are mean \pm SEM of $n=6-7,{ }^{*} \mathrm{p}<0.05$ vs EOC TC.

Estrogen pre-stimulated EOCs decrease migration but not proliferation of hCASMCs. We showed that eNOS overexpressing EOCs regulate hCASMC migration (Fig. 3a). To determine whether $\mathrm{E}_{2}$-stimulated EOCs could have a similar effect, EOCs were pre-stimulated with $\mathrm{E}_{2}$, rinsed, and incubated with 
a
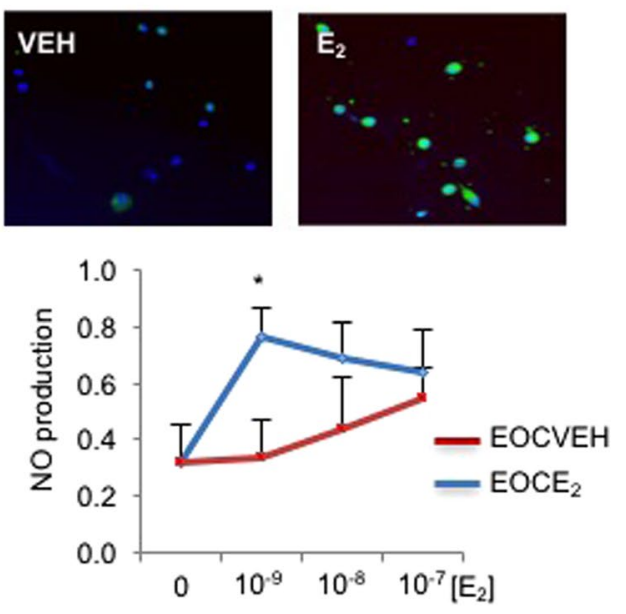

b

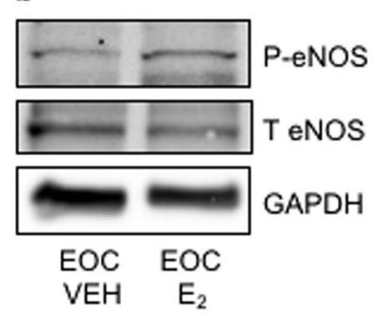

c

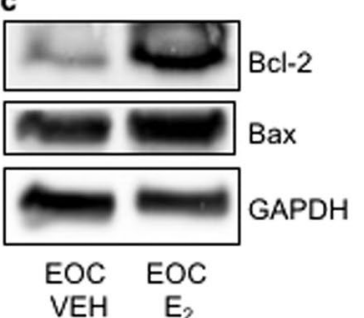

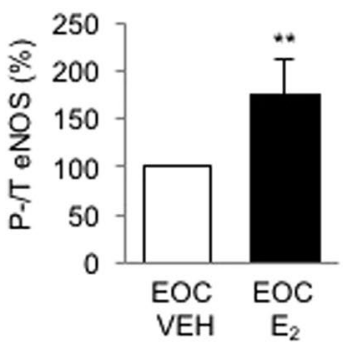

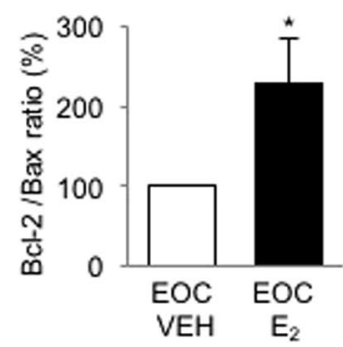

Figure 4. Estrogen increases eNOS phosphorylation, NO production and survival of EOCs. Human EOCs were stimulated with vehicle (VEH) or estrogen $\left(\mathrm{E}_{2}\right)$ at different concentrations $(\mathbf{a})$ or at $10^{-9} \mathrm{M}(\mathbf{b}, \mathbf{c})$. (a) Nitric oxide (NO) production by EOCs was measured by DAF-FM diacetate, a NO fluorescent probe (green). Results are expressed as DAF-FM/Dapi positive cell ratios reported to untreated cells. Representative images of EOCs stimulated with VEH or $\mathrm{E}_{2}\left(10^{-9} \mathrm{M}\right)$ (right). (b) Total (T) and phosphorylated (pS1177) (P) eNOS expression was evaluated by western blot. (c) Cell survival was assessed by Bcl-2 and Bax protein expression ratio by western blot. Data are mean \pm SEM of $n=6,{ }^{*} p<0.05$ and $* * p<0.01$ vs VEH.

a

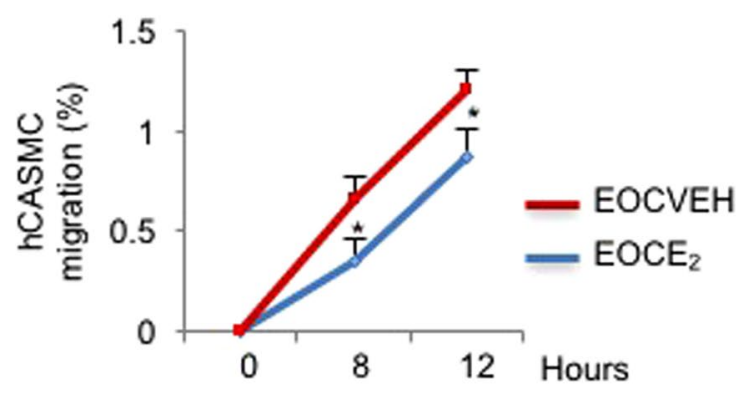

b

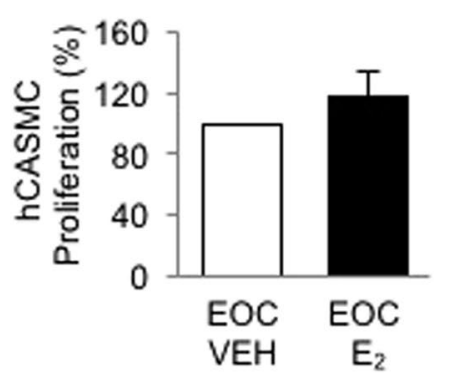

Figure 5. Estrogen pre-stimulated EOCs decrease migration but not proliferation of SMCs. Human EOCs were pre-stimulated with vehicle (VEH) or estrogen $\left(\mathrm{E}_{2}, 10^{-9} \mathrm{M}\right)$ for $24 \mathrm{~h}$ and co-cultured with human coronary artery smooth muscle cells for up to 12 hours (a) or 24 hours (b). (a) SMC migration was assessed by scratch assay. (b) SMC proliferation was measured by Ki67 immunostaining. Data are mean \pm SEM of $n=6,{ }^{*} p<0.05$ vs VEH (at same time point).

the $\mathrm{E}_{2}$ receptor inhibitor fulvestrant $\left(10^{-9} \mathrm{M}\right)$ prior to co-culture with hCASMCs. We found a significant decrease of hCASMC migration in the presence of $\mathrm{E}_{2}$-stimulated EOCs compared with vehicle (Fig. 5a). However, we did not observe an effect on hCASMC proliferation (Fig. 5b). These results suggest that hCASMC migration could be regulated by EOCs pre-treated with $\mathrm{E}_{2}$.

eNOS transfection and $E_{2}$ stimulation have an additive effect on NO production and cell apoptosis in EOCs. To determine whether there is an additive effect of eNOS transfection and $\mathrm{E}_{2}$ stimulation in EOCs, cells were transfected with eNOS plasmid and stimulated with $\mathrm{E}_{2}$. Interestingly, we observed a higher NO production in EOCs with both treatments as compared to only $\mathrm{E}_{2}$ - stimulated EOCs. In contrast, there was no further increase in eNOS phosphorylation (Fig. 6a,b). This was associated with a further decrease of active caspase 3 expression (Fig. 6c), without any effect on Bcl-2/ Bax ratio (Fig. 6d). These results suggest that there is a partially additive effect of eNOS transfection and $\mathrm{E}_{2}$ treatment on NO production and cell survival in EOCs.

eNOS transfection and $E_{2}$ stimulation of EOCs do not have an additive effect on hCASMC migration and proliferation. To determine whether there is an additive effect of eNOS transfection and $\mathrm{E}_{2}$ stimulation on hCASMC migration or proliferation, EOCs were transfected with eNOS plasmid and stimulated 
a

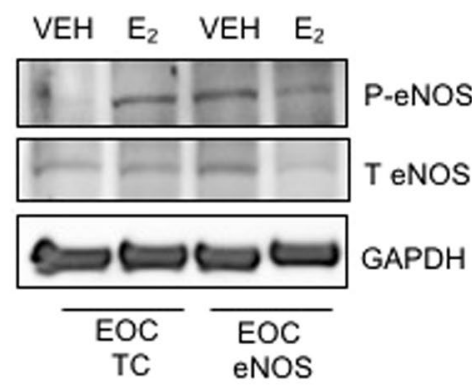

c

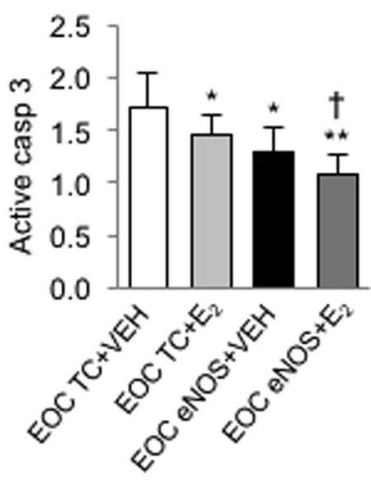

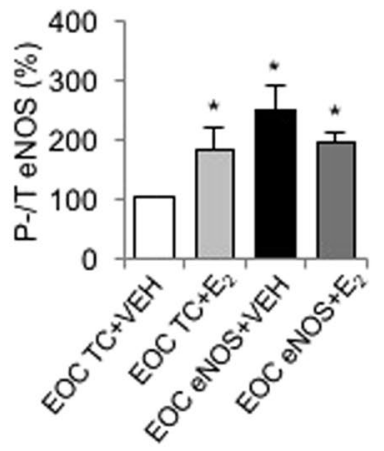

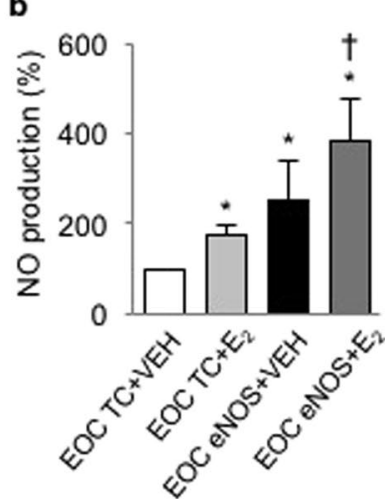

d
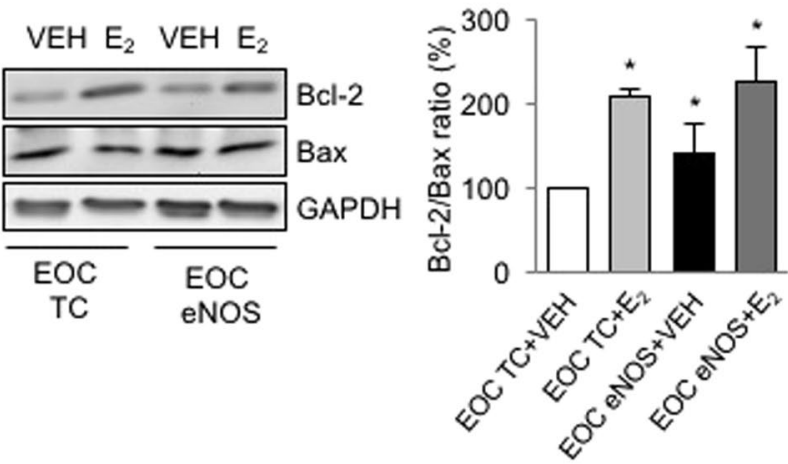

Figure 6. eNOS upregulation by plasmid transfection and estrogen have an additive effect on NO production and apoptosis reduction in EOCs. Human EOCs were transfected with a transfection control plasmid (EOC TC) or eNOS plasmid (EOC eNOS) and stimulated with vehicle (VEH, DMSO) or estrogen $\left(\mathrm{E}_{2}, 10^{-9} \mathrm{M}\right)$ for 24 hours. (a) Total (T) and phosphorylated (P) eNOS (S1177) expression was evaluated by western blot. (b) Nitric oxide (NO) production was assessed by DAF-FM, a NO fluorescent probe. (c) Apoptosis was measured by active caspase 3 flow cytometry. (d) Cell survival was assessed by Bcl-2 and Bax protein expression ratio by western blot. Data are mean \pm SEM of $\mathrm{n}=6,{ }^{*} \mathrm{p}<0.05$ and $* * \mathrm{p}<0.01$ vs EOC TC $+\mathrm{VEH}, \uparrow \mathrm{p}<0.05$ vs EOC $\mathrm{TC}+\mathrm{E}_{2}$.

with $\mathrm{E}_{2}$ before being co-cultured with hCASMCs. We did not observe any supplemental effect of combined treatments on hCASMC migration or proliferation (Fig. 7). These results indicate that despite an increased NO production in EOCs both transfected with eNOS and treated with $\mathrm{E}_{2}$, there is no further effect on hCASMC function.

Intact or eNOS-transfected EOCs do not have any effect on ERK1/2 or FAK phosphorylation in hCASMCs. To investigate the molecular mechanism involved in the inhibitory effect of eNOS-transfected or $\mathrm{E}_{2}$-stimulated EOCs on hCASMC function, we investigated FAK and ERK1/2 phosphorylation, two pathways involved in cell migration and proliferation. EOCs were transfected with eNOS or transfection control plasmid, or were stimulated with $\mathrm{E}_{2}$, or received both treatments prior to co-culture with hCASMCs. In some experiments, platelet-derived growth factor (PDGF, $10^{-7} \mathrm{M}$ ) was added at the time of co-culture.

A basal level of FAK phosphorylation was observed in SMCs, and no further effect of PDGF was noted (Supplemental Figs 1-3). In comparison, ERK1/2 activity was increased in SMCs stimulated with PDGF. We did not observe any effect of EOC co-culture on hCASMC FAK or ERK1/2 phosphorylation. Moreover, neither eNOS transfection nor $\mathrm{E}_{2}$ stimulation of EOCs, nor the two combined, had any impact on SMC FAK or ERK1/2 signaling. These results suggest that the inhibitory effect of eNOS overexpressing EOCs on hCASMC migration is not through FAK or ERK1/2 pathways.

\section{Discussion}

This study demonstrates that human eNOS-overexpressing EOCs have increased survival rate and demonstrate improved functionality through paracrine effects. Frequently termed "endothelial progenitor cells", circulating EOCs are defined according to surface markers and properties. One recent classification distinguishes EOCs, which display mostly paracrine actions, from late outgrowth cells, which are characterized by high proliferative potential ${ }^{23}$. EOCs are derived from the bone marrow and are involved in postnatal vasculogenesis and reendothelialization after endothelial damage $e^{24,25}$. EOCs have been used as a therapeutic tool in multiple clinical studies, but the therapeutic benefit of these cells is attenuated by age and disease $\mathrm{e}^{26,27}$. Even in the absence of such mitigating factors, our data demonstrates that naïve EOCs have little influence on SMC migration or proliferation in 


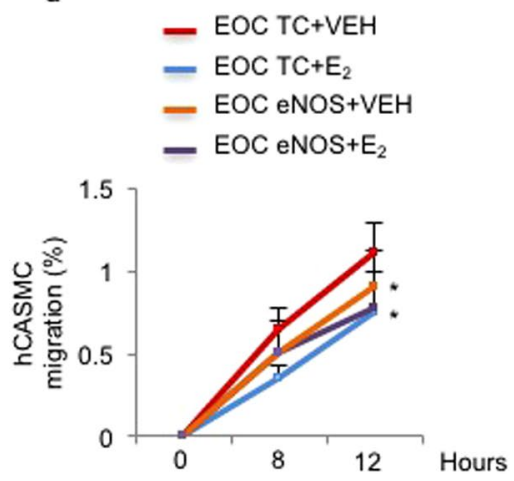

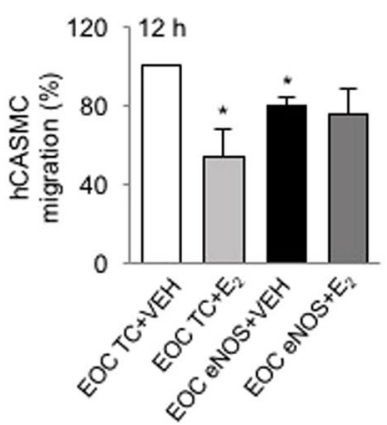

b

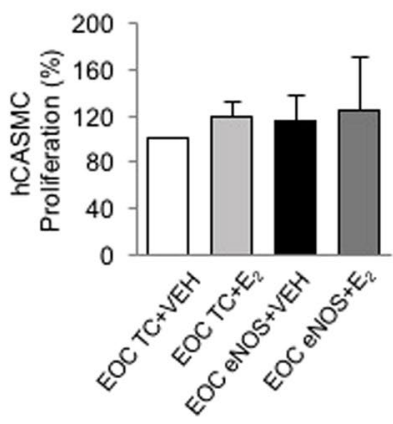

Figure 7. eNOS upregulation by plasmid transfection and estrogen do not have any additive effect on SMC migration and proliferation in EOCs. Human EOCs were transfected with a transfection control plasmid (EOC TC) or eNOS plasmid (EOC eNOS) or and stimulated with vehicle (VEH) or estrogen $\left(\mathrm{E}_{2}, 10^{-9} \mathrm{M}\right)$ for 24 hours. They were then co-cultured with human coronary artery smooth muscle cells for up to 12 hours (a) or 24 hours (b). (a) SMC migration was assessed by scratch assay at 8 and 12 hours. (b) SMC proliferation was measured by Ki67 immunostaining. Data are mean \pm SEM of $n=6, * p<0.05$ vs EOC TC + VEH.

vitro, but that increasing eNOS production by EOCs through transfection or estrogen stimulation significantly enhances their effects, particularly as relates to curtailing SMC migration.

$\mathrm{NO}$ is a well-known anti-proliferative and pro-survival factor and its effects on SMC proliferation and migration are extensively reported in the literature ${ }^{28}$. Therefore, the use of EOCs engineered to produce more NO was a logical step to improve effectiveness of these cells. Overexpression of eNOS or exogenous estrogen has previously been shown to enhance the capacity of bone marrow-derived EOCs to abate neointimal hyperplasia ${ }^{7,29-31}$. This protective effect was attributed to enhanced re-endothelialization, but no effects on SMCs were considered. Gene transfer of eNOS in bone-marrow derived mesenchymal stem cells was also found to improve cardiac repair after myocardial infarction ${ }^{32}$. Similarly, in models of pulmonary arterial hypertension, injection of fibroblasts ${ }^{33}$, mesenchymal stem cells ${ }^{34}$, or bone marrow-derived endothelial-like progenitor cells ${ }^{35}$ transfected with eNOS improved recovery, compared with null plasmid transfected-cells which allowed the disease to progress. Hence, many studies report a reparative effect of EOCs in models of vascular disease, but how these cells act on underlying SMCs has been poorly investigated.

For one, the magnified effects of eNOS-overexpressing EOCs be contingent on their increased viability. There is indeed evidence that the beneficial effects of some drugs, including estrogen, may hinge on NO-dependent reduced senescence and apoptosis of EOCs ${ }^{29-31}$. Depending on the dosage and the presence or not of stimulatory co-signals, NO has been shown to block or to induce apoptosis ${ }^{36}$. In stem cells, NO may induce auto-protection by decreasing the activity of caspase 3 through direct interaction ${ }^{37}$. The increase in EOC survival by eNOS/ NO pathway stimulation could be clinically very relevant since one of the critical limitations for the therapeutic application of postnatal EOCs is their low number in the circulation, which is even lower in patients with cardiovascular risk factors ${ }^{38}$. In this regard, we evaluated the effect of eNOS stimulation and overexpression on EOC survival. Following estrogen treatment or eNOS plasmid transfection, total and phosphorylated eNOS levels, as well as NO production, were increased in EOCs. We observed a significant decrease in cleaved caspase 3, accompanied by an enhanced $\mathrm{Bcl}-2 / \mathrm{Bax}$ ratio in these cells compared with controls. Combining eNOS transfection and estrogen treatment significantly increased NO production and reduced caspase 3 activity. Hence, the results suggest that in our experimental conditions, enhancing NO reduces EOC apoptosis.

Thereafter, we investigated the potential effects of native, estrogen-stimulated and eNOS over-expressing EOCs on SMC function. We first confirmed that the NO donor SNAP inhibited proliferation and migration of vascular SMCs in a dose dependent manner, in line with previous studies ${ }^{39-41}$. We then found that co-culture of SMCs with native EOCs did not affect SMC proliferation or migration, but it did sensitize the cells to SNAP, reducing the concentration of the NO donor required to abate SMC migration and proliferation. Although Cui et al. showed in vivo that transplantation of native EOCs reduced neointimal hyperplasia in denuded rat carotid arteries $^{7}$, our results suggest that in vitro, native EOC NO production is not sufficient to regulate the activity of neighboring cells. The next step was to investigate the effect of NO-overproducing EOCs on SMC migration and proliferation. Interestingly, we observed a significant decrease in migration but not proliferation in SMC co-cultured both with estrogen-stimulated and with eNOS-transfected EOCs. In support of our results, multiple NO donors were reported to inhibit SMC migration in a concentration-dependent, reversible, and non-cytotoxic fashion $^{42}$. However, the absence of effect on SMC proliferation suggests that higher NO levels might be required to modulate SMC proliferation in vitro. Indeed, Mooradian et al. studied the effect of different NO donors on SMC growth and showed that the inhibitory effect depended on the total amount of NO delivered, the rate of delivery, and the period of exposure to $\mathrm{NO}^{43}$. In our experiments, even combining estrogen treatment and eNOS overexpression failed to meet the minimum threshold required for inhibition of proliferation.

We further investigated the molecular pathway involved in the inhibitory effect observed on SMC migration by EOCs. Platelet-derived growth factor (PDGF) is a potent inducer of SMC proliferation and migration, 
frequently involved in neointima formation ${ }^{44}$. It acts by binding to its receptor that initiates a cascade of signals leading to the activation of the ERK pathway ${ }^{45}$. PDGF receptors have been shown to be localized to focal adhesions and stimulate focal adhesion kinase (FAK), which is linked not only to activation of $\mathrm{ERK}^{46}$ but also to integrin-dependent SMC migration ${ }^{47}$. PDGF-dependent SMC proliferation and migration is reduced by $\mathrm{NO}^{48}$, and recent data have revealed that even native EOCs were shown to attenuate the response of SMCs to PDGF ${ }^{49}$. We investigated ERK1/2 and FAK phosphorylation in SMCs co-cultured with native, estrogen-stimulated, or eNOS-transfected EOCs. Under basal conditions, EOCs did not have any effect on ERK1/2 or FAK phosphorylation, suggesting that even in the absence of PDGF the inhibitory effects of enhanced EOCs on SMC migration are unlikely to have involved FAK. Furthermore, PDGF activated ERK1/2 in SMCs, and the presence of any EOCs did not abolish this effect. Thus, not only did EOCs fail to reduce SMC proliferation in basal conditions, but even in conditions of accentuated SMC proliferation (with PDGF), EOCs are unlikely to have an effect.

Many vascular diseases are exacerbated by uncontrolled SMC proliferation and migration, including restenosis after angioplast ${ }^{50}$ and pulmonary hypertension ${ }^{51}$. In such conditions, strategies have been devised to optimize the therapeutic potential of EOCs. Hence, it was reported that angioplasty stents coated with anti-CD34 antibodies, designed to capture circulating EOCs, induced the rapid establishment of a functional endothelial layer early in the damaged area with minimal inflammation ${ }^{24}$. More relevant to the current work, eNOS-overexpressing EOCs were recently found to be well tolerated in patients with pulmonary arterial hypertension, as part of a first phase trial to establish the clinical applicability of such a strategy ${ }^{20}$. Thus, understanding to what extent eNOS-transfected EOCs can act on SMCs is a priority.

This study comprises some limitations, however. For one, we did not uncover the mechanism whereby estrogen-stimulated or eNOS-overexpressing EOCs repress SMC migration, other than to rule out FAK and ERK signaling. This is surprising, given that FAK and integrin activation have long been associated with SMC migration $^{52,53}$. Another potential pathway to consider is the Rho kinase signaling cascade, recently shown to be activated in SMCs in a model of pulmonary hypertension, and to be sensitive to eNOS-activating treatment ${ }^{51}$. Another point to consider is that SMCs contribute to atherosclerotic plaque stability ${ }^{53}$, and that limiting their migration may impact vulnerability. Nevertheless, this could be offset by integration of EOCs themselves into atherosclerotic lesions, participating in vascular repair ${ }^{54}$.

In summary, here we show for the first time that $\mathrm{NO}$ overproducing EOCs have a better survival and decrease SMC migration through paracrine effects. These data are potentially clinically relevant since they determine the potential of primed EOCs to prevent pathological vascular remodeling.

\section{Methods}

All methods were carried out in accordance with relevant guidelines and regulations.

Mononuclear cell isolation and EOC characterization. In order to isolate human EOCs, peripheral blood was obtained from healthy volunteers, (age 20-45 years, male and female, no known disease or medication). All experimental protocols including blood cell donation were approved by the research review committee of the Lady Davis Institute for Medical Research. Informed consent was obtained from all donors. EOCs derived from male and female donors produced comparable results, even in estrogen stimulation experiments. Hence, cells from both genders were pooled.

The $\mathrm{n}$ value is the number of times each experiment was repeated. Each individual experiment was performed with a distinct EOC population grown from cells isolated from one individual.

Twenty $\mathrm{ml}$ citrate-buffered blood was mixed with $15 \mathrm{ml}$ of phosphate buffered saline. Then, $15 \mathrm{ml}$ ficoll (Sigma-Aldrich) was carefully overlaid with the diluted blood and centrifuged for 20 minutes. The mononuclear cells (MNCs) found in the interphase were carefully collected. MNCs $\left(5 \times 10^{5}\right)$ were seeded on fibronectin-coated plates in endothelial basal medium (EBM) with supplements (EGM-2, Lonza), as previously described ${ }^{9}$.

After 7 days of culture, EOCs were characterized as previously described ${ }^{9}$. Fibronectin-adherent cells were stained with 1,1'-dioctadecyl- 3,3,3',3' tetramethylindocarbocyanine (Dil)-labeled acetylated low-density lipoprotein (acLDL; Invitrogen) and FITC-labeled lectin from Ulex europaeus agglutinin (Sigma). Cells double-positive for Dil-acLDL and lectin staining were considered to be early outgrowth cells. Quantitative immunocytochemical analysis ${ }^{55}$ revealed that Dil-acLDL/lectin double-positive cells express the endothelial markers VEGF receptor- 2 $(100 \pm 0 \%)$, phospho-endothelial NOS $(100 \pm 0 \%)$, CD31 $(84 \pm 8 \%)$ and to some degree the stem cell marker CD34 (18 $\pm 1 \%)$.

EOC transfection. On day 4 of culture MNCs were transfected according to the following protocol: $3 \mu \mathrm{l}$ of X-tremeGENE 9 transfection reagent (Roche) were mixed for every $100 \mu$ of EBM (Lonza). Plasmid DNA was then added in a 3:1 ratio ( $\mu$ l of transfection reagent: $\mu$ g of plasmid DNA) and incubated for 15 min at room temperature. The transfection complex was added in a dropwise manner on the cell culture in a 1:10 volumetric ratio (transfection complex:cell medium). Cells were transfected with either dialyzed pVAX1-heNOS plasmid DNA or pmaxFP-Green-C (Lonza) plasmid as a transfection control. Cells were cultured for $48 \mathrm{~h}$ after transfection.

$17 \beta$-estradiol stimulations. On day 6 of culture MNCs were treated with $1-100 \mathrm{nM} \mathrm{E}_{2}$ (Sigma-Aldrich) for 30 minutes (for time course experiments). To prevent hCASMC $\mathrm{E}_{2}$ derived stimulation in the EOC- hCASMC co-culture setting; $\mathrm{E}_{2}$ treated EOCs were previously washed with PBS, placed in an incubator for $30 \mathrm{~min}$ with $10 \mathrm{nM}$ of the estrogen receptor inhibitor fulvestrant (Sigma-Aldrich) followed by another wash prior the co-culture.

Western blotting. Cultured EOCs were harvested with a cell lysis buffer (HEPES $10 \mathrm{nM} \mathrm{pH} \mathrm{7.4,} \mathrm{Na} \mathrm{pyroph-}$ osphate $50 \mathrm{nM}, \mathrm{NaF} 50 \mathrm{nM}, \mathrm{Na}_{2}$ EDTA $5 \mathrm{nM}$, EGTA $5 \mathrm{nM}$, Triton X-100 0.5\%, $\mathrm{Na}_{3} \mathrm{VO}_{4} 2 \mathrm{nM}$, PMSF $1 \mathrm{nM}$, leupeptin $1 \mu \mathrm{g} / \mathrm{ml}$, aprotinin $1 \mu \mathrm{g} / \mathrm{ml}$ ). Protein levels were quantified by Bradford assay (Bio-Rad). The appropriate 
volume of SDS loading buffer (375 mM Tris- $\mathrm{HCl} \mathrm{pH} \mathrm{6.8,} \mathrm{6 \%} \mathrm{SDS,} \mathrm{48 \%} \mathrm{glycerol,} \mathrm{9 \%} \mathrm{2-mercaptoethanol,} 0.03 \%$ bromophenol blue) was added to protein samples, heated for $5 \mathrm{~min}$ at $100^{\circ} \mathrm{C}$ and loaded into $8 \%$ polyacrylamide gel. Proteins were transferred overnight to a PVDF membrane (Bio-Rad). The membrane was blocked for 1 hour at room temperature with blocking buffer (5\% non-fat dry milk in TBS-T (50 mM Tris pH 7.6, $150 \mathrm{mM} \mathrm{NaCl}$, Tween-20 0.05\%)) and then incubated overnight with the appropriate primary antibodies. The membrane was washed 3 times with TBS-T and incubated with the appropriate HRP secondary antibodies (Santa Cruz goat anti-rabbit or goat anti-mouse). Next the membrane was incubated with a western lightning plus-ECL solution (Perkin Elmer). The chemiluminescence was measured using Chemidoc XRS + system (Bio-Rad) and quantified by densitometry using Quantity One software (Bio-Rad).

BrdU assay. Primary human coronary artery smooth muscle cells (hCASMC) (PromoCell) were grown to $\sim 70 \%$ confluency in EGM-2. After $24 \mathrm{~h}$ serum starving, BrdU reagent (EMD Millipore) was added at 1/3000 final concentration and cell culture inserts (BD Biosciences) containing 1 million EOCs were immediately placed over the hCASMC. After 24 hs of co-culture, EOCs were discarded; hCASMC were incubated with a fixing solution following an incubation with $1 / 200 \mathrm{BrdU}$ detector antibody for 1 hour and then with a goat anti-mouse IgG peroxidase conjugate. Cells were then washed as described and incubated with $200 \mu \mathrm{l}$ per well of TMB peroxidase substrate. The luminescence was measured at $450 \mathrm{~nm}$ with a fluorescence plate reader (BMG Labtech FLUOstar Optima).

Ki-67 staining. hCASMC were seeded at an appropriate density on cover slips (Fisher Scientific). Cells were fixed with $2 \%$ paraformaldehyde and permeabilized with $0.5 \%$ Triton X-100 for $10 \mathrm{~min}$. Cells were blocked with $3 \%$ BSA overnight at $4{ }^{\circ} \mathrm{C}$, then rinsed with PBS and incubated with $1 / 50$ diluted rabbit anti-Ki-67 antibody (Abcam) for $90 \mathrm{~min}$ followed by a fluorescent secondary antibody (Invitrogen). Images were taken using a Leica DM2000 fluorescent microscope (Leica Microsystems) and an Infinity3-1UC color camera (Leica Microsystems). Ki-67 positive cells were quantified as a percentage of total DAPI stained nuclei and normalized with either untreated EOCs or transfection control EOCs (basal conditions). For each experimental condition, an average of 200 cells were counted from 3-4 microscopic fields, and $\sim 15 \%$ cells in each field were Ki-67+.

Wound scratch assay. hCASMC were seeded in a 24 well plate in EGM-2 medium. After a 24-hour starving cells were wounded once with a small tip by scratching across the maximum diameter of each well. Pictures were taken immediately after scratching (time 0 ). Cell culture inserts containing EOCs were placed over the hCASMC. 30 minutes later, $0.1 \mu \mathrm{M}$ PDGF (R\&D Systems) or the nitric oxide donor, S-nitroso-N-acetylpenicillamine (SNAP, Sigma Aldrich) was added as needed. pictures were also taken at 8 and 12 hours with EOC co-culture. Images were analyzed using Image J software by measuring the size of the denuded area.

Flow cytometry. Cells were incubated with human FcR blocking reagent (Miltenyi Biotec), then fixed and permeabilized (BD Cytofix/Cytoperm Fixation/Permeabilization). Cleaved caspase-3 antibody (Cell Signaling) was added at a concentration of 1/50. Cleaved caspase- 3 positive cells were quantified using a FACSCalibur flow cytometer. Results were analyzed using CellQuest Pro software (BD Biosciences).

NO probe. MNCs were seeded on fibronectin coated cover slips. On day 6, DAF-FM diacetate (Molecular Probes) was added for 1 hour at $37^{\circ} \mathrm{C}$. Then, cells were fixed with $2 \%$ paraformaldehyde. Cover slips were mounted on slides using a mounting medium containing DAPI. Images were taken using an Infinity 3 Leica fluorescent microscope. NO-positive cells were quantified as ratios of DAF-DM/Dapi positive cells and normalized with untreated EOCs (for E2 time course experiments) and expressed as percentage of EOCTC (for eNOS overexpressing experiments). A single threshold level was applied to all experiments and all conditions for analysis of DAF-FM levels (ImageJ). For each experimental condition, an average of 265 cells were counted from 4 microscopic fields.

Statistical Analysis. All data are presented as mean \pm standard error of the mean (SEM). We performed a Student's t-test to compare two groups, or a two-way ANOVA followed by the Newman-Keuls post-test for multiple group comparisons. Probability values of $\mathrm{p} \leq 0.05$ were considered to be statistically significant.

\section{References}

1. Davignon, J. \& Ganz, P. Role of endothelial dysfunction in atherosclerosis. Circulation 109, III27-32 (2004).

2. Ross, R. Atherosclerosis-an inflammatory disease. N. Engl. J. Med. 340, 115-126 (1999).

3. Foteinos, G., Hu, Y., Xiao, Q., Metzler, B. \& Xu, Q. Rapid endothelial turnover in atherosclerosis-prone areas coincides with stem cell repair in apolipoprotein E-deficient mice. Circulation 117, 1856-1863 (2008).

4. Asahara, T. et al. Bone marrow origin of endothelial progenitor cells responsible for postnatal vasculogenesis in physiological and pathological neovascularization. Circ. Res. 85, 221-228 (1999).

5. Walter, D. H., Zeiher, A. M. \& Dimmeler, S. Effects of statins on endothelium and their contribution to neovascularization by mobilization of endothelial progenitor cells. Coron. Artery Dis. 15, 235-242 (2004).

6. Werner, N. et al. Bone marrow-derived progenitor cells modulate vascular reendothelialization and neointimal formation: effect of 3-hydroxy-3-methylglutaryl coenzyme a reductase inhibition. Arterioscler. Thromb. Vasc. Biol. 22, 1567-1572 (2002).

7. Cui, B. et al. Transplantation of endothelial progenitor cells overexpressing endothelial nitric oxide synthase enhances inhibition of neointimal hyperplasia and restores endothelium-dependent vasodilatation. Microvasc. Res. 81, 143-150 (2011).

8. Miller-Kasprzak, E. \& Jagodziński, P. P. Endothelial progenitor cells as a new agent contributing to vascular repair. Arch. Immunol. Ther. Exp. (Warsz.) 55, 247-259 (2007).

9. Ebrahimian, T. et al. Inhibition of four-and-a-half LIM domain protein-2 increases survival, migratory capacity, and paracrine function of human early outgrowth cells through activation of the sphingosine kinase-1 pathway: implications for endothelial regeneration. Circ. Res. 114, 114-123 (2014).

10. Sambuceti, G. et al. Diabetes impairs the vascular recruitment of normal stem cells by oxidant damage, reversed by increases in pAMPK, heme oxygenase-1, and adiponectin. Stem Cells. 27, 399-407 (2009). 
11. Ruifrok, W.-P. T. et al. Estradiol-induced, endothelial progenitor cell-mediated neovascularization in male mice with hind-limb ischemia. Vasc. Med. 14, 29-36 (2009).

12. Haynes, M. P. et al. Membrane estrogen receptor engagement activates endothelial nitric oxide synthase via the PI3-kinase-Akt pathway in human endothelial cells. Circ. Res. 87, 677-682 (2000).

13. Walter, D. H. et al. Statin therapy accelerates reendothelialization: a novel effect involving mobilization and incorporation of bone marrow-derived endothelial progenitor cells. Circulation 105, 3017-3024 (2002).

14. Lantin-Hermoso, R. L. et al. Estrogen acutely stimulates nitric oxide synthase activity in fetal pulmonary artery endothelium. Am. J. Physiol. 273, L119-126 (1997).

15. Caulin-Glaser, T., García-Cardeña, G., Sarrel, P., Sessa, W. C. \& Bender, J. R. 17 beta-estradiol regulation of human endothelial cell basal nitric oxide release, independent of cytosolic Ca2+ mobilization. Circ. Res. 81, 885-892 (1997).

16. MacRitchie, A. N. et al. Estrogen upregulates endothelial nitric oxide synthase gene expression in fetal pulmonary artery endothelium. Circ. Res. 81, 355-362 (1997).

17. Landmesser, U. et al. Statin-induced improvement of endothelial progenitor cell mobilization, myocardial neovascularization, left ventricular function, and survival after experimental myocardial infarction requires endothelial nitric oxide synthase. Circulation 110, 1933-1939 (2004).

18. Iwakura, A. et al. Estrogen-mediated, endothelial nitric oxide synthase-dependent mobilization of bone marrow-derived endothelial progenitor cells contributes to reendothelialization after arterial injury. Circulation 108, 3115-3121 (2003).

19. Urao, N. et al. Erythropoietin-mobilized endothelial progenitors enhance reendothelialization via Akt-endothelial nitric oxide synthase activation and prevent neointimal hyperplasia. Circ. Res. 98, 1405-1413 (2006).

20. Granton, J. et al. Endothelial NO-Synthase Gene-Enhanced Progenitor Cell Therapy for Pulmonary Arterial Hypertension: The PHACeT Trial. Circ. Res. 117, 645-654 (2015).

21. Quyyumi, A. A. et al. PreSERVE-AMI: A Randomized, Double-Blind, Placebo-Controlled Clinical Trial of Intracoronary Administration of Autologous CD34+ Cells in Patients with Left Ventricular Dysfunction Post STEMI. Circ. Res. 120, 324-331 (2016).

22. Zhao, Y., Vanhoutte, P. M. \& Leung, S. W. S. Vascular nitric oxide: Beyond eNOS. J. Pharmacol. Sci. 129, 83-94 (2015).

23. Minami, Y. et al. Angiogenic potential of early and late outgrowth endothelial progenitor cells is dependent on the time of emergence. Int. J. Cardiol. 186, 305-314 (2015).

24. Lim, W.-H. et al. Stent coated with antibody against vascular endothelial-cadherin captures endothelial progenitor cells, accelerates re-endothelialization, and reduces neointimal formation. Arterioscler. Thromb. Vasc. Biol. 31, 2798-2805 (2011).

25. Koller, L. et al. Prognostic relevance of circulating endothelial progenitor cells in patients with chronic heart failure. Thromb. Haemost. 116, 309-316 (2016).

26. Hu, J. et al. Novel Targets of Drug Treatment for PulmonaryHypertension. Am. J. Cardiovasc. Drugs Drugs Devices Interv. 15, 225-234 (2015).

27. Rurali, E. et al. BM ageing: Implication for cell therapy with EPCs. Mech. Ageing Dev. 159, 4-13 (2016).

28. Lei, J., Vodovotz, Y., Tzeng, E. \& Billiar, T. R. Nitric oxide, a protective molecule in the cardiovascular system. Nitric Oxide Biol. Chem. 35, 175-185 (2013).

29. Liu, Y., Wei, J., Hu, S. \& Hu, L. Beneficial effects of statins on endothelial progenitor cells. Am. J. Med. Sci. 344, 220-226 (2012).

30. Strehlow, K. et al. Estrogen increases bone marrow-derived endothelial progenitor cell production and diminishes neointima formation. Circulation 107, 3059-3065 (2003).

31. Iwakura, A. et al. Estradiol enhances recovery after myocardial infarction by augmenting incorporation of bone marrow-derived endothelial progenitor cells into sites of ischemia-induced neovascularization via endothelial nitric oxide synthase-mediated activation of matrix metalloproteinase-9. Circulation 113, 1605-1614 (2006).

32. Chen, L., Zhang, Y., Tao, L., Yang, Z. \& Wang, L. Mesenchymal Stem Cells with eNOS Over-Expression Enhance Cardiac Repair in Rats with Myocardial Infarction. Cardiovasc. Drugs Ther. 31, 9-18 (2017).

33. Zhao, Y. D. et al. Microvascular regeneration in established pulmonary hypertension by angiogenic gene transfer. Am. J. Respir. Cell Mol. Biol. 35, 182-189 (2006).

34. Kanki-Horimoto, S. et al. Implantation of mesenchymal stem cells overexpressing endothelial nitric oxide synthase improves right ventricular impairments caused by pulmonary hypertension. Circulation 114, I181-185 (2006).

35. Zhao, Y. D. et al. Rescue of monocrotaline-induced pulmonary arterial hypertension using bone marrow-derived endothelial-like progenitor cells: efficacy of combined cell and eNOS gene therapy in established disease. Circ. Res. 96, 442-450 (2005).

36. Shen, Y. H., Wang, X. L. \& Wilcken, D. E. Nitric oxide induces and inhibits apoptosis through different pathways. FEBS Lett. 433, 125-131 (1998)

37. Kim, Y. M., Talanian, R. V. \& Billiar, T. R. Nitric oxide inhibits apoptosis by preventing increases in caspase-3-like activity via two distinct mechanisms. J. Biol. Chem. 272, 31138-31148 (1997).

38. Al Mheid, I. et al. Age and Human Regenerative Capacity Impact of Cardiovascular Risk Factors. Circ. Res. 119, 801-809 (2016).

39. Cornwell, T. L., Arnold, E., Boerth, N. J. \& Lincoln, T. M. Inhibition of smooth muscle cell growth by nitric oxide and activation of cAMP-dependent protein kinase by cGMP. Am. J. Physiol. 267, C1405-1413 (1994).

40. Ignarro, L. J. et al. Role of the arginine-nitric oxide pathway in the regulation of vascular smooth muscle cell proliferation. Proc. Natl. Acad. Sci. USA 98, 4202-4208 (2001).

41. Dubey, R. K., Jackson, E. K. \& Lüscher, T. F. Nitric oxide inhibits angiotensin II-induced migration of rat aortic smooth muscle cell. Role of cyclic-nucleotides and angiotensin1 receptors. J. Clin. Invest. 96, 141-149 (1995).

42. Sarkar, R., Meinberg, E. G., Stanley, J. C., Gordon, D. \& Webb, R. C. Nitric oxide reversibly inhibits the migration of cultured vascular smooth muscle cells. Circ. Res. 78, 225-230 (1996).

43. Mooradian, D. L., Hutsell, T. C. \& Keefer, L. K. Nitric oxide (NO) donor molecules: effect of NO release rate on vascular smooth muscle cell proliferation in vitro. J. Cardiovasc. Pharmacol. 25, 674-678 (1995).

44. Kohno, T. et al. IQGAP1 links PDGF receptor- $\beta$ signal to focal adhesions involved in vascular smooth muscle cell migration: role in neointimal formation after vascular injury. Am. J. Physiol. 305, C591-600 (2013).

45. Graf, K. et al. Mitogen-activated protein kinase activation is involved in platelet-derived growth factor-directed migration by vascular smooth muscle cells. Hypertension. 29, 334-339 (1997).

46. Zhang, F. et al. The matricellular protein Cyr61 is a key mediator of platelet-derived growth factor-induced cell migration. J. Biol. Chem. 290, 8232-8242 (2015).

47. Ishigaki, T. et al. Tenascin-C enhances crosstalk signaling of integrin $\alpha v \beta 3 / P D G F R-\beta$ complex by SRC recruitment promoting PDGF-induced proliferation and migration in smooth muscle cells. J. Cell. Physiol. 226, 2617-2624 (2011).

48. Seymour, K., Han, X., Sadowitz, B., Maier, K. G. \& Gahtan, V. Differential effect of nitric oxide on thrombospondin-1-, PDGF- and fibronectin-induced migration of vascular smooth muscle cells. Am. J. Surg. 200, 615-619 (2010).

49. Wang, H. et al. Transplantation of EPCs overexpressing PDGFR- $\beta$ promotes vascular repair in the early phase after vascular injury. BMC Cardiovasc. Disord. 16, 179-191 (2016).

50. Marx, S. O., Totary-Jain, H. \& Marks, A. R. Vascular smooth muscle cell proliferation in restenosis. Circ. Cardiovasc. Interv. 4, 104-111 (2011)

51. Lee, M.-Y. et al. Liraglutide prevents and reverses monocrotaline-induced pulmonary arterial hypertension by suppressing ET-1 and enhancing eNOS/sGC/PKG pathways. Sci. Rep. 6, 31788 (2016). 
52. Mehta, P. K. \& Griendling, K. K. Angiotensin II cell signaling: physiological and pathological effects in the cardiovascular system. Am. J. Physiol. 292, C82-97 (2007).

53. Finney, A. C., Stokes, K. Y., Pattillo, C. B. \& Orr, A. W. Integrin signaling in atherosclerosis. Cell. Mol. Life Sci. 74, 2263-2282 (2017).

54. Yu, B., Chen, Q., Le Bras, A., Zhang, L. \& Xu, Q. Vascular Stem/Progenitor Cell Migration and Differentiation in Atherosclerosis. Antioxid. Redox Signal., https://doi.org/10.1089/ars.2017.7171 (2017).

55. Endtmann, C. et al. Angiotensin II impairs endothelial progenitor cell number and function in vitro and in vivo: implications for vascular regeneration. Hypertension. 58, 394-403 (2011).

\section{Acknowledgements}

The authors wish to thank Sven Wassmann and Jean-Francois Tanguay for fruitful discussions. David Courtman kindly supplied the eNOS and control plasmids used in these experiments. This study was supported by the Canadian Foundation for Innovation (CFI), Canada Research Chairs (CRC), the Canadian Institutes of Health Research (CIHR), and the Fonds de recherche du Quebec - Santé (FRQS)/ThéCell.

\section{Author Contributions}

S.L. designed the study. S.G. and T.E. performed the experiments. S.L., T.E. and M.H. wrote the manuscript. T.E. prepared the figures. N.E. helped with study design and reviewed the manuscript.

\section{Additional Information}

Supplementary information accompanies this paper at https://doi.org/10.1038/s41598-017-18848-z.

Competing Interests: The authors declare that they have no competing interests.

Publisher's note: Springer Nature remains neutral with regard to jurisdictional claims in published maps and institutional affiliations.

(c) (i) Open Access This article is licensed under a Creative Commons Attribution 4.0 International

License, which permits use, sharing, adaptation, distribution and reproduction in any medium or format, as long as you give appropriate credit to the original author(s) and the source, provide a link to the Creative Commons license, and indicate if changes were made. The images or other third party material in this article are included in the article's Creative Commons license, unless indicated otherwise in a credit line to the material. If material is not included in the article's Creative Commons license and your intended use is not permitted by statutory regulation or exceeds the permitted use, you will need to obtain permission directly from the copyright holder. To view a copy of this license, visit http://creativecommons.org/licenses/by/4.0/.

(c) The Author(s) 2018 